\begin{tabular}{l|l|l|l|l}
\hline Volume 2 & Issue 3 & December (2021) & DOI: 10.47540/ijsei.v2i3.398 & Page: $258-270$ \\
\hline
\end{tabular}

\title{
Annotated Checklist and Conservation Status of Mammal Species in Sarpang District, Bhutan
}

\author{
Jigme Tenzin ${ }^{1}$, Phub Dhendup ${ }^{2}$, Dago Dorji ${ }^{3}$, Sangay Nidup ${ }^{4}$, Phuntsho Thinley ${ }^{5}$ \\ ${ }^{1-5}$ Department of Forests and Park Services, Ministry of Agriculture \& Forests, Bhutan \\ Corresponding Author: Jigme Tenzin; Email: jigmetenzin16@gmail.com
}

\begin{tabular}{|c|c|}
\hline A R T I C & E I N F O \\
\hline $\begin{array}{l}\text { Keywords } \\
\text { Species; I } \\
\text { Monitorin }\end{array}$ & $\begin{array}{l}\text { Globally Threatened } \\
\text { ventory; Mammal Spec }\end{array}$ \\
\hline Received & : 14 November 2021 \\
\hline Revised & : 24 December 2021 \\
\hline Accepted & : 25 December 2021 \\
\hline
\end{tabular}

\section{LE INFO}

Keywords: Globally Threatened Species; Inventory; Mammal Species;

Received : 14 November 2021

Accepted : 25 December 2021

\begin{abstract}
A B S T R A C T
Bhutan has a total geographical area of $38,394 \mathrm{Km}^{2}$ located in between the IndoMalayan and Palearctic region, out of which $51.44 \%\left(19750.75 \mathrm{~km}^{2}\right)$ of its total geographical area has been designated as the protected area. However, none of the districts have a structured baseline checklist of mammal species documented till date. Therefore, Sarpang Forest Division under the Department of Forests and Park Services had carried out five rigorous camera trap surveys including a nationwide tiger survey that covers representable areas of the district from 2014 till 2020. The survey shows that district has 36 mammal species that belong to 18 families under seven orders. Felidae and Cervidae families has the highest species abundance $(\mathrm{n}=$ 17\%), while, Canidae, Herpestidae, Leporidae, Manidae, Melinae, Muridae, Mustelidae, Tupaiidae, Proboscidae, Pteromyidae, Suidae and Ursidae were the lowest $(\mathrm{n}=3 \%)$. Above all, Sarpang homed $29.90 \%$ of total mammal species of Bhutan, out of which 3\% of mammal species were categorized under Critically Endangered, 14\% Endangered, 14\% Vulnerable, 22\% Near Threatened, and 47\% Least Concern as per IUCN Red List. However, only 20 mammal species are listed under CITES and nine in Schedule I of Forest and Nature Conservation Act of Bhutan, 1995. Therefore, landscape-based planning such as the Division-based Conservation \& Management plan; periodic monitoring of wildlife species using camera traps, and validation of Schedule I species are suggested for long-term conservation and management of globally threatened species inside the landscape of Sarpang district in Bhutan.
\end{abstract}

\section{INTRODUCTION}

Bhutan has a total geographical area of 38,394 $\mathrm{Km}^{2}$ located in between the two ecological regions: The Indo-Malayan region and Palearctic region (Wangchuck et al., 2004; Tenzin et al., 2019). Due to this landscape's convergence and persistence guidance from a farsighted monarch, has leads to designate $51.44 \%$ of total geographical areas into the protected area (PA) that harbors more than 200 mammal species protected by the sound conservation policies (Wangchuk et al., 2004; Dhendup and Dorji, 2018). Thus, Bhutan is included within the landscape of Himalayan biodiversity hotspots (Myers et al., 2000) and Global 200 ecoregions (Olsen and Dinerstein, 2002; Mitermeier et al., 2004).

Ecologically, mammal plays an important role in ecosystems which provides numerous essential ecosystem services such as seed dispersal, pollination and regulating insect populations, and reducing disease transmissions (Keesing et al., 2010; Kunz et al., 2011) and some also evidence as an indicator of ecosystem health (Jones et al., 2009). However, rapid declines in mammalian biodiversity (Schipper, 2008; Penjor et al., 2021) were induced by the monopolization of ecosystems and natural resources by anthropogenic activities (Mace et al., 2005; Butchart et al., 2011; Penjor et al., 2021). On other hand, the lack of data and climate change effects (high temperature and evapotranspiration in tropical areas) also exacerbate the species decline across the globe (Jones and Safi, 2011; Jones and Safi, 2011; Penjor et al., 2021). Further, the studies had also predicted that by 2070 , the impact of land-use change is predicted to globally endanger $\sim 1700$ species of amphibians, 
birds and mammals, including species of high conservation value and functional importance, due to habitat contraction (Barlow et al., 2016; Powers and Jetz, 2019; Penjor et al., 2021).

The latest nationwide on-site camera traps survey has recorded only 129 mammal species listed in the Biodiversity Statistics of Bhutan (NBC, 2017; NCD, 2020). On other hand, entire national parks and wildlife sanctuaries under the Department of Forests and Park Services (DoFPS) have their own mammal checklists attached with their Conservation Management Plans. While some have separate printed checklist books as well as published scientific papers. For instance, Jomotsangkha Wildlife Sanctuary [JWS], Phibsoo Wildlife Sanctuary [PWS], Jigme Dorji National Park [JDNP], and Jigme Khesar Strict Nature Reserve [JKSNR] have a separate printed checklist of mammal's books that has recorded $34,36,33 \&$ 41 species of mammals respectively (JWS, 2018; PWS, 2019; Koirala and Jamtsho, 2019; JKSNR, 2020). Thus, the existence of a structured mammal checklist in the protected areas (PAs) has not only helped in developing pragmatic management plans but also ensures in developing effective conservation policies (Esmaeili et al., 2017).

However, none of the Non-Protected Areas (NPAs) managed by 14 Divisional Forests Offices (DFOs) under DoFPS and districts have structured checklist of mammal species published till date. The DFOs were established long before 1957 which is before the inception of the Protected Areas management system in Bhutan (Forest Resource Management Division [FRMD], 2019). Nationwide Tiger survey of 1989 was the first-ever scientific study in Bhutan, that covers both PAs and NPAs including Sarpang district (Dorji and Santiapillai, 1989), followed by second (McDougal and Tshering, 1998)\& third nationwide tiger survey in 2014-2015 (DoFPS, 2015). Later, nationwide elephant's survey in 2016; selective tiger monitoring survey in 2018, and rapid biodiversity assessment [RBA]inside \& outside Biological Corridor-03 [BC-03] in 2019 have covered representable areas of Sarpang district (Tenzin et al., 2021). However, the district still lacks comprehensive inventories of mammal species except for a few scanty studies on felid species by Tenzin et al. (2019) and Tenzin et al. (2021) in the light of rapidly changing ecosystems in Bhutan (Dhendup and Dorji 2018; Penjor et al., 2018). Therefore, the paper aims to document first-ever checklists, determine relative species abundance, and conservation status of mammal species through consolidation of past camera traps data (2014-2020) to facilitate periodic monitoring and management of wildlife species under the jurisdiction of Sarpang district in Bhutan.

\section{MATERIALS AND METHOdS \\ Study area}

Sarpang district has a total geographical area of $1,655.37 \mathrm{Km}^{2}$ located in between $26^{\circ} 52^{\prime}$ North and $90^{\circ} 16^{\prime}$ East (Ministry of Work \& Human Settlement [MoWHS], 2019) in the southern central part of Bhutan (Figure 1). The district falls within the convergences of three ecologically-diverse protected areas (RMNP, JSWNP, and PWS) of Bhutan, connected to each other by BC-03 (Tenzin et al., 2021). The district shares the southern border with the Northeast state of Assam, India which has further connected their landscapes with the Royal Manas National Park [RMNP] and Indian Manas National Park [MNP] towards the east. While PWS in the west connects with Buxa Tiger Reserve [BTR] in West Bengal. Thus, the entire integration of RMNP, MNP, PWS, and BTR landscapes has holistically formed one of the biggest tiger conservation landscapes called, "Northern Forest Complex-Namdhapha-Royal Manas (NFC-N-RM)" in Eastern Himalayas (Tempa, 2017; Tempa et al., 2019; Tenzin et al., 2021). 


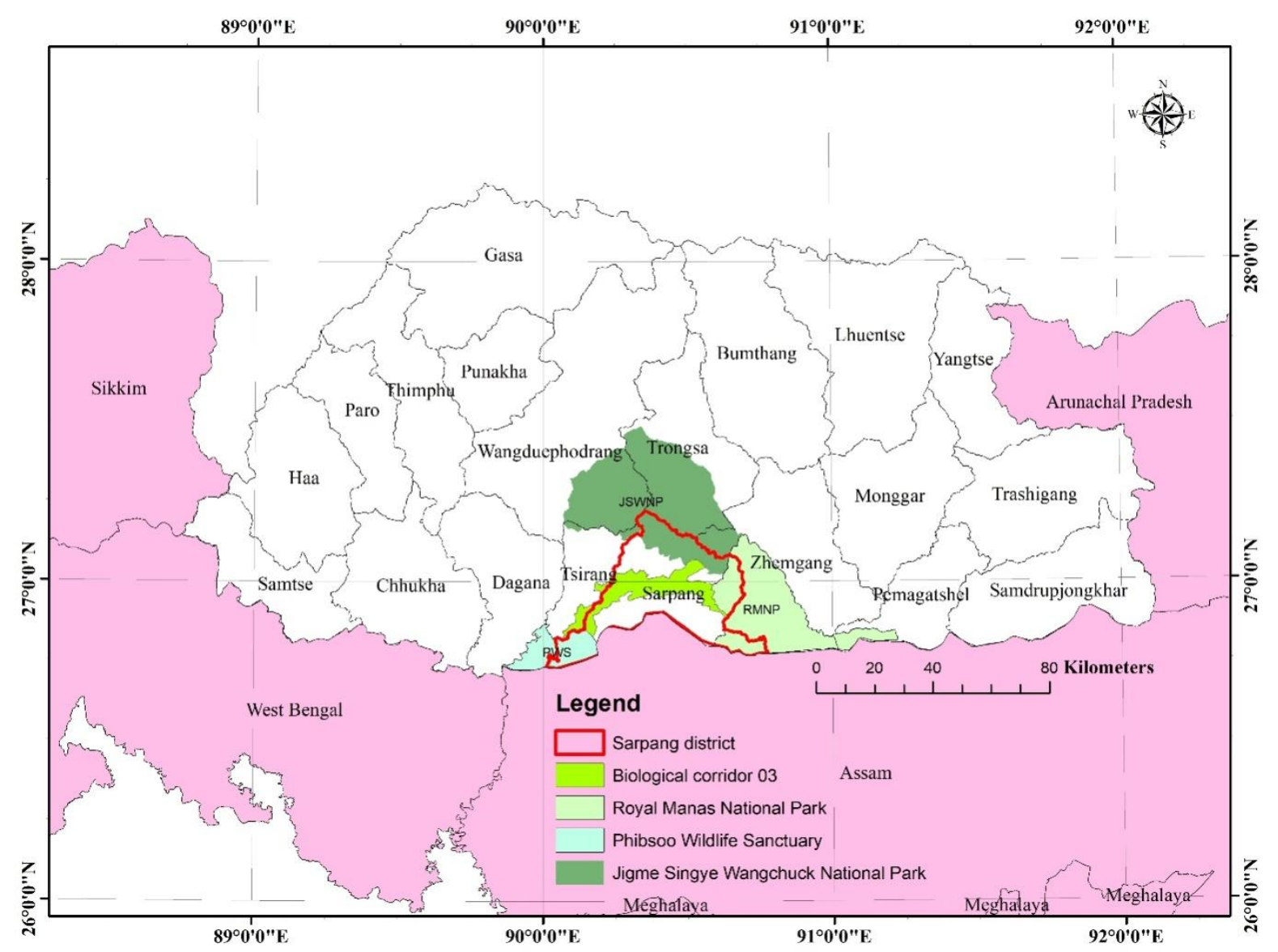

Figure 1. Bhutan map showing the location of Sarpang district (red outline) in between the three ecologically diverse protected connected each other by Biological corridor-03 in the south-central part of Bhutan.

Elevation ranges from 153 to 3,506 masl (Tenzin et al., 2018) with annual precipitation of $3,500-5,500 \mathrm{~mm}$ (DOA, 2012). The district has a diverse forest type: sub-tropical broadleaved (153$1000)$, warm broad forest (1000-2500), and cool broadleaved forest (2500-3000) (Oshawa, 1987). Sarpang district alone has a total population of 46,004 that lives in 10,369 households across the 12 blocks (NSB, 2018).

\section{Data collection}

\section{Camera Traps}

Data were collected using a camera trap of Nationwide Tiger Survey [NTS] (2014-2015); National Elephants survey (2016); selective tiger monitoring camera trap survey (2018), RBA inside BC-03 (2019), and Rapid assessment of tiger and prey habitats (2020), which together had covered representable area of the Sarpang district. A total of 70 camera traps were stationed inside 35 grids (grid sizes of $5 \times 5 \mathrm{~km}$ ) under Sarpang during NTS (2014-2015). While, 11 camera traps were selectively stationed inside NTS grids in 2018 and another 37 camera traps (grid sizes of $4 \times 4 \mathrm{~km}$ ) during the recent RBA and Conservation Assured Tiger Standards [CA|TS] survey (Tenzin et al., 2019; Tenzin et al., 2021). In the case of NTS, two cameras/grid were stationed along trails for the periods of seven months (DoFPS, 2015), while, one camera traps/grid were used during RBA, selective tiger monitoring, and CA|TS survey respectively for the period of three months due to limited camera traps and budgets. Meanwhile, cameras like Reconyx, Cuddle back, U-way, and Scout guard were used and stationed $45-50 \mathrm{~cm}$ above the ground.

\section{Field Observation}

On-site photographs of mammal species captured from the jurisdiction of Sarpang Forest Division were also included in this mammal checklists. Species were identified using mammals of Bhutan (Wangchuck et al., 2004) and Mammals of the Indian sub-continent (Menon, 2012). Relative 
species abundance and conservation status of each species as per International Union for Conservation of Nature [IUCN], Convention on International Trade in Endangered Species of Wild Fauna and Flora [CITES] and Forest and Nature Conservation Act of Bhutan [FNCAB] (1995) were also been provided for better information.

\section{Data analysis}

Data of entire camera traps images were sorted, segregated, and analyzed using Renamer software (Sanderson and Harris, 2012) and generated the mammal checklists. While the determination of relative species abundance and updation of conservation status were carried out using the Pivot table of MS excel 2016.

\section{RESULTS AND DISCUSSION}

Sarpang Forest Division (SFD) had carried out five extensive division-wide camera traps survey which had recorded a total of 36 mammal species that belong to 18 families under seven orders (Table $1 \& 2$ ).

Table 1. Lists of mammal species and its distribution range in Bhutan.

\begin{tabular}{|c|c|c|c|}
\hline Sl.\# & Scientific Name & Distribution Range & Source \\
\hline & $\begin{array}{l}\text { Order Rodent } \\
\text { Family: Hystricidae }\end{array}$ & & \\
\hline 1 & $\begin{array}{l}\text { Atherurus macrourus, Linnaeus, } 1758 \\
\text { Asiatic Brush-tailed porcupine }\end{array}$ & PWS, RMNP, Sarpang & $\begin{array}{l}\text { Wangchuk et al., 2004; PWS, } \\
2019 .\end{array}$ \\
\hline 2 & $\begin{array}{l}\text { Hysterix bracyhura, Linnaeus, } 1758 \\
\text { Himalayan crestless porcupine }\end{array}$ & $\begin{array}{l}\text { JKSNR, JWS, PWS and } \\
\text { Sarpang }\end{array}$ & $\begin{array}{l}\text { Koirala and Jamtsho 2019; PWS, } \\
\text { 2019; JKSNR, } 2020 .\end{array}$ \\
\hline & $\begin{array}{l}\text { Order Carnivora } \\
\text { Family: Felidae }\end{array}$ & & \\
\hline 3 & $\begin{array}{l}\text { Catopuma temmincki, Vigors and } \\
\text { Horsfield, } 1827 \\
\text { Asiatic golden cat }\end{array}$ & $\begin{array}{l}\text { RMNP, JSWNP, JKSNR, } \\
\text { PWS, Sarpang and Gedu } \\
\text { (Chukha) }\end{array}$ & $\begin{array}{l}\text { Tempa et al., 2011; Dorji et el., } \\
\text { 2017; } \\
\text { Koirala and Jamtsho, 2019; PWS, } \\
\text { 2019; Tenzin et al., 2019; } \\
\text { JKSNR, 2020. }\end{array}$ \\
\hline 4 & $\begin{array}{l}\text { Neofelis nebulosa, Griffith } 1821 \\
\text { Clouded leopard }\end{array}$ & $\begin{array}{l}\text { JSWNP, JKSNR, } \\
\text { Gedu(Chukha), RMNP and } \\
\text { Sarpang }\end{array}$ & $\begin{array}{l}\text { Tempa et al., 2011; Koirala and } \\
\text { Jamtsho, 2019. Penjor et al., } \\
\text { 2019, PWS, 2019; Tenzin et al., } \\
\text { 2019; }\end{array}$ \\
\hline 5 & $\begin{array}{l}\text { Panthera pardus, Linnaeus, } 1758 \\
\text { Common leopard }\end{array}$ & $\begin{array}{l}\text { JSWNP, JKSNR, PNP, } \\
\text { RMNP also from Sarpang }\end{array}$ & $\begin{array}{l}\text { Tempa et al., 2011; Koirala and } \\
\text { Jamtsho 2019; Tenzin et al., } \\
\text { 2019; JKSNR, } 2020 .\end{array}$ \\
\hline 6 & $\begin{array}{l}\text { Prionailurus bengalensis, Kerr } 1792 \\
\text { Leopard cat }\end{array}$ & $\begin{array}{l}\text { BWS, JSWNP, JKSNR, } \\
\text { RMNP and also from } \\
\text { Sarpang. }\end{array}$ & $\begin{array}{l}\text { Tempa et al., 2011; Koirala and } \\
\text { Jamtsho 2019; Tenzin et al., } \\
\text { 2019; JKSNR, 2020. }\end{array}$ \\
\hline 7 & $\begin{array}{l}\text { Parofelis marmorata, Martim } 1837 \\
\text { Marbled cat }\end{array}$ & $\begin{array}{l}\text { RMNP, Lamaigonpa } \\
\text { (Bumthang), JDNP, PWS, } \\
\text { JKSNR also from Sarpang. }\end{array}$ & $\begin{array}{l}\text { Tempa et al., 2011; Koirala and } \\
\text { Jamtsho, 2019; Tenzin et al., } \\
\text { 2019; Dhendup and Tenzin, } \\
\text { 2020; JKSNR, 2020. }\end{array}$ \\
\hline \multirow[t]{2}{*}{8} & $\begin{array}{l}\text { Panthera tigris, Linnaeus, } 1758 \\
\text { Tiger }\end{array}$ & $\begin{array}{l}\text { Sarpang, Zhemgang, Gasa, } \\
\text { Trongsa, Bumthang, } \\
\text { Trashigang and JKSNR, } \\
\text { JDNP and PWS }\end{array}$ & $\begin{array}{l}\text { Wangchuk et al., 2004; DoFPS, } \\
\text { 2015; Koirala and Jamtsho, 2019; } \\
\text { Tenzin et al., 2019; JKSNR, } \\
2020 .\end{array}$ \\
\hline & $\begin{array}{l}\text { Order: Carnivora } \\
\text { Family: Canidae }\end{array}$ & & \\
\hline \multirow[t]{2}{*}{9} & $\begin{array}{l}\text { Cuon alpinus primaevus Pallas, } 181 \\
\text { Wild dog }\end{array}$ & $\begin{array}{l}\text { JDNP, JKSNR, JSWNP, } \\
\text { JWS, PWS, RMNP and } \\
\text { Sarpang }\end{array}$ & $\begin{array}{l}\text { Wangchuk et al., 2004; JWS, } \\
\text { 2018; PWS, 2019; Koirala and } \\
\text { Jamtsho, 2019; JKSNR, } 2020 .\end{array}$ \\
\hline & $\begin{array}{l}\text { Order Carnivora } \\
\text { Family: Ursidae }\end{array}$ & & \\
\hline 10 & Ursus thibetanus laniger, Cuvier 1823 & JDNP, JKSNR, PWS, JWS, & Wangchuk et al., 2004; JWS, \\
\hline
\end{tabular}




\begin{tabular}{|c|c|c|c|}
\hline & Himalayan black bear & $\begin{array}{l}\text { Thimphu and Paro \& } \\
\text { Sarpang }\end{array}$ & $\begin{array}{l}\text { 2018; Koirala and Jamtsho, 2019; } \\
\text { PWS, 2019; JKSNR, 2020. }\end{array}$ \\
\hline & $\begin{array}{l}\text { Order Proboscidea } \\
\text { Family: Elephantidae }\end{array}$ & & \\
\hline \multirow[t]{2}{*}{11} & $\begin{array}{l}\text { Elephas maximus, Linnaeus, } 1756 \\
\text { Asian elephant }\end{array}$ & $\begin{array}{l}\text { Samtse, Sarpang, Samdrup } \\
\text { Jongkhar, Lhamoizhingkha } \\
\text { (Dagana), JWS, PWS, } \\
\text { RMNP. }\end{array}$ & $\begin{array}{l}\text { Wangchuk et al., 2004; Nature } \\
\text { Conservation Division [NCD], } \\
\text { 2018; PWS, } 2019 .\end{array}$ \\
\hline & $\begin{array}{l}\text { Order Artiodactyla } \\
\text { Family: Cervidae }\end{array}$ & & \\
\hline 12 & $\begin{array}{l}\text { Muntiacus mutjak, Zimmermann, } \\
1780 \text { Barking deer }\end{array}$ & $\begin{array}{l}\text { Distribution recorded across } \\
\text { Bhutan }\end{array}$ & $\begin{array}{l}\text { Wangchuk et al., 2004; JWS, } \\
\text { 2018; Koirala and Jamtsho, 2019; } \\
\text { PWS, 2019; JKSNR, 2020. }\end{array}$ \\
\hline 13 & $\begin{array}{l}\text { Bos gaurus, C.H. Smith, } 1827 \\
\text { Guar }\end{array}$ & $\begin{array}{l}\text { RMNP, JWS, PWS, } \\
\text { JKSNR, Bangtar (Samdrup } \\
\text { Jongkhar), Nganglam } \\
\text { (Pemagatshel), Sarpang. }\end{array}$ & $\begin{array}{l}\text { Wangchuk et al., 2004; JWS, } \\
\text { 2018; Koirala and Jamtsho, 2019; } \\
\text { JKSNR, } 2020 .\end{array}$ \\
\hline 14 & $\begin{array}{l}\text { Nemorhaedus goral, Hardwicke, } 1825 \\
\text { Himalayan goral }\end{array}$ & $\begin{array}{l}\text { JDNP, JWS, PWS, RMNP, } \\
\text { Rimchu (Gasa), Sarpang }\end{array}$ & $\begin{array}{l}\text { Wangchuk et al., 2004; Koirala } \\
\text { and Jamtsho, 2019; PWS, 2019; } \\
\text { JKSNR, 2020. }\end{array}$ \\
\hline 15 & $\begin{array}{l}\text { Capricornis sumatraensis, Hodgson, } \\
1831 \\
\text { Himalayan serow }\end{array}$ & $\begin{array}{l}\text { Deothang (Samdrup } \\
\text { Jongkhar), RMNP, } \\
\text { Khebethang(Wangdue), } \\
\text { Sakteng (Tashigang), } \\
\text { Barshong (Tsirang), } \\
\text { Lingzhi, JDNP, PWS, } \\
\text { JWS\& Sarpang. }\end{array}$ & $\begin{array}{l}\text { Wangchuk et al., 2004; JWS, } \\
\text { 2018; Koirala and Jamtsho, 2019; } \\
\text { JKSNR, } 2020 .\end{array}$ \\
\hline 16 & $\begin{array}{l}\text { Cervus unicolor, Kerr, } 1792 \\
\text { Sambar }\end{array}$ & $\begin{array}{l}\text { RMNP, JSWNP, JWS, } \\
\text { SWS, JDNP, PNP } \\
\text { \&Sarpang }\end{array}$ & $\begin{array}{l}\text { Wangchuk et al., 2004; JWS, } \\
\text { 2018; Koirala and Jamtsho, } \\
\text { 2019; JKSNR, } 2020 .\end{array}$ \\
\hline \multirow[t]{2}{*}{17} & $\begin{array}{l}\text { Axis porcinus, Zimmermann, } 1780 \\
\text { Hog deer }\end{array}$ & $\begin{array}{l}\text { RMNP, PWS JWS, Singye, } \\
\text { Serzhong, and Gelephu } \\
\text { (Sarpang). }\end{array}$ & Wangchuk et al., 2004 \\
\hline & $\begin{array}{l}\text { Order Artiodactyla } \\
\text { Family: Suidae }\end{array}$ & & \\
\hline \multirow[t]{2}{*}{18} & $\begin{array}{l}\text { Sus scrofa, Linnaeus, } 1758 \\
\text { wild pig }\end{array}$ & $\begin{array}{l}\text { Distribution recorded across } \\
\text { Bhutan including Sarpang. }\end{array}$ & $\begin{array}{l}\text { Wangchuk et al., 2004; Koirala } \\
\text { and Jamtsho, 2019; JWS, 2018; } \\
\text { PWS, 2019; JKSNR, 2020. }\end{array}$ \\
\hline & $\begin{array}{l}\text { Order Rodentia } \\
\text { Family: Sciuridae }\end{array}$ & & \\
\hline 19 & $\begin{array}{l}\text { Ratufa bicolor, Sparrman, } 1778 \\
\text { Malayan giant squirrel }\end{array}$ & $\begin{array}{l}\text { JKSNR, JDNP, JWS and } \\
\text { Sarpang }\end{array}$ & $\begin{array}{l}\text { JWS, 2018; Koirala and Jamtsho, } \\
\text { 2019; JKSNR, } 2020 .\end{array}$ \\
\hline 20 & $\begin{array}{l}\text { Dremomys lokriah, Hodgson, } 1836 \\
\text { Orange-bellied squirrel }\end{array}$ & $\begin{array}{l}\text { JDNP, JKSNR and } \\
\text { Sarpang }\end{array}$ & $\begin{array}{l}\text { Koirala and Jamtsho, 2019; } \\
\text { JKSNR, } 2020 .\end{array}$ \\
\hline 21 & $\begin{array}{l}\text { Callosciurus pygerythrus, Geoffroy } \\
\text { Saint-Hilaire, } 1831 \\
\text { Hoary-bellied squirrel }\end{array}$ & $\begin{array}{l}\text { Distribution recorded from } \\
\text { Sarpang }\end{array}$ & \\
\hline \multirow[t]{2}{*}{22} & $\begin{array}{l}\text { Callosciurus erythraeus, Pallas, } 1799 \\
\text { Pallas squirrel }\end{array}$ & JDNP, JKSNR and Sarpang & $\begin{array}{l}\text { Koirala and Jamtsho, 2019; } \\
\text { JKSNR, } 2020 .\end{array}$ \\
\hline & $\begin{array}{l}\text { Order Primates } \\
\text { Family: Cercopithecoidea }\end{array}$ & & \\
\hline 23 & $\begin{array}{l}\text { Macaca assamensis, M'clelland, } 1840 \\
\text { Assamese macaque }\end{array}$ & $\begin{array}{l}\text { Distribution recorded in } \\
\text { between Thimphu- }\end{array}$ & $\begin{array}{l}\text { Wangchuk et al., 2004; Koirala } \\
\text { and Jamtsho, 2019; PWS, 2019; }\end{array}$ \\
\hline
\end{tabular}




\begin{tabular}{|c|c|c|c|}
\hline & & $\begin{array}{l}\text { Phuntsholing, Punakha, } \\
\text { Trongsa, Zhemgang, } \\
\text { Trashigang, JDNP, JKSNR, } \\
\text { PWS, and Sarpang }\end{array}$ & JKSNR, 2020. \\
\hline 24 & $\begin{array}{l}\text { Macaca mulatta, Zimmermann, } 1780 \\
\text { Rhesus macaque }\end{array}$ & $\begin{array}{l}\text { RMNP and PWS and } \\
\text { Sarpang. }\end{array}$ & $\begin{array}{l}\text { Wangchuk et al., 2004; JWS, } \\
\text { 2018; PWS, 2019. }\end{array}$ \\
\hline \multirow[t]{2}{*}{25} & $\begin{array}{l}\text { Trachypithecus geei, Khajuria, } 1956 \\
\text { Golden langur }\end{array}$ & $\begin{array}{l}\text { Trongsa, Zhemgang, } \\
\text { Gelephu(Sarpang), RMNP, } \\
\text { PWS and Tsirang }\end{array}$ & $\begin{array}{l}\text { Wangchuk et al., 2004; Thinley } \\
\text { et al., 2019; PWS, } 2019 .\end{array}$ \\
\hline & $\begin{array}{l}\text { Order Primates } \\
\text { Family: Lorissidae }\end{array}$ & & \\
\hline \multirow[t]{2}{*}{26} & $\begin{array}{l}\text { Nycticebus bengalensis, Lacepede, } \\
1800 \\
\text { Bengal slow glories }\end{array}$ & $\begin{array}{l}\text { RMNP, Jigmeling, Dekiling } \\
\text { and Samtenling (Sarpang) }\end{array}$ & $\begin{array}{l}\text { Wangchuk et al., 2004; Thinley } \\
\text { et al., } 2019 .\end{array}$ \\
\hline & $\begin{array}{l}\text { Order Carnivora } \\
\text { Family: Mustelidae }\end{array}$ & & \\
\hline \multirow[t]{2}{*}{27} & $\begin{array}{l}\text { Martes flavigula, Bodaert, } 1785 \\
\text { Yellow-throated marten }\end{array}$ & $\begin{array}{l}\text { Distribution recorded } \\
\text { throughout Bhutan } \\
\text { including Sarpang }\end{array}$ & $\begin{array}{l}\text { Wangchuk et al., 2004; Koirala } \\
\text { and Jamtsho, 2019;PWS, 2019; } \\
\text { JKSNR, 2020. }\end{array}$ \\
\hline & $\begin{array}{l}\text { Order Carnivora } \\
\text { Family: Viverridae }\end{array}$ & & \\
\hline 28 & $\begin{array}{l}\text { Paguma larvata, C.E.H.Smith, } 1827 \\
\text { Himalayan palm civet }\end{array}$ & $\begin{array}{l}\text { Namling(Mongar), JDNP, } \\
\text { and hilly area of Sarpang }\end{array}$ & Wangchuk et al., 2004 \\
\hline 29 & $\begin{array}{l}\text { Viverra zibetha, Linnaeus, } 1758 \\
\text { Large Indian civet }\end{array}$ & $\begin{array}{l}\text { Langthel (Trongsa), } \\
\text { JKSNR, JDNP, PWS and } \\
\text { Sarpang }\end{array}$ & $\begin{array}{l}\text { Wangchuk et al., 2004; PWS, } \\
\text { 2019; Koirala and Jamtsho, } \\
\text { 2019; JKSNR, } 2020 .\end{array}$ \\
\hline \multirow[t]{2}{*}{30} & $\begin{array}{l}\text { Viverricula indica, } \\
\text { Geoffroy Saint-Hilaire, } 1803 \\
\text { Small Indian civet }\end{array}$ & $\begin{array}{l}\text { RMNP and PWS and } \\
\text { Sarpang. }\end{array}$ & Wangchuck et al., 2004 \\
\hline & $\begin{array}{l}\text { Order Carnivora } \\
\text { Family: Herpestidae }\end{array}$ & & \\
\hline \multirow[t]{2}{*}{31} & $\begin{array}{l}\text { Herpestes urva, Hodgson, } 1836 \\
\text { Crab eating mongoose }\end{array}$ & $\begin{array}{l}\text { RMNP, PWS, JDNP and } \\
\text { JKSNR and Sarpang }\end{array}$ & $\begin{array}{l}\text { Wangchuk et al., 2004; Koirala } \\
\text { and Jamtsho, 2019; PWS, 2019; } \\
\text { JKSNR, 2020. }\end{array}$ \\
\hline & $\begin{array}{l}\text { Order Rodentia } \\
\text { Family: Muridae }\end{array}$ & & \\
\hline 32 & $\begin{array}{l}\text { Mus musculus, Linnaeus, } 1758 \\
\text { House mouse }\end{array}$ & $\begin{array}{l}\text { Distribution recorded from } \\
\text { Sarpang }\end{array}$ & \\
\hline \multirow[t]{2}{*}{33} & $\begin{array}{l}\text { Mus Pahari, Thomas, } 1916 \\
\text { Sikkim mouse }\end{array}$ & $\begin{array}{l}\text { Distribution recorded from } \\
\text { Sarpang }\end{array}$ & \\
\hline & $\begin{array}{l}\text { Order Carnivora } \\
\text { Family: Mustelidae }\end{array}$ & & \\
\hline \multirow[t]{2}{*}{34} & $\begin{array}{l}\text { Arctonyx collaris, Cuvier, } 1825 \\
\text { Hog badger }\end{array}$ & RMNP including Sarpang & Wangchuk et al., 2004 \\
\hline & $\begin{array}{l}\text { Order Legomorpha } \\
\text { Family: Leporidae }\end{array}$ & & \\
\hline \multirow[t]{2}{*}{35} & $\begin{array}{l}\text { Lepus nigricollis, F.Cuvier, } 1823 \\
\text { Indian hare }\end{array}$ & $\begin{array}{l}\text { Distribution recorded from } \\
\text { southern foothills including } \\
\text { Sarpang }\end{array}$ & Wangchuk et al., 2004 \\
\hline & $\begin{array}{l}\text { Order Pholidota } \\
\text { Family: Manidae }\end{array}$ & & \\
\hline 36 & $\begin{array}{l}\text { Manis pentadactyla, Linnaeus, } 1758 \\
\text { Chinese pangolin }\end{array}$ & $\begin{array}{l}\text { RMNP and Samdrup } \\
\text { Jongkhar, Pelrithang jail }\end{array}$ & Wangchuk et al., 2004 \\
\hline
\end{tabular}




\begin{tabular}{|l|l|l|}
\hline & $\begin{array}{l}\text { area, and Gelephu } \\
\text { (Sarpang) }\end{array}$ & \\
\hline
\end{tabular}

Note: JDNP: Jigme Dorji National Park; RMNP: Royal Manas National Park; JSWNP: Jigme Singye Wangchuck National Park; PNP: Phrumsengla National Park; PWS: Phibsoo Wildlife Sanctuary; JWS: Jomotsangkha Wildlife Sanctuary.

\section{Relative Species Abundances}

Relative species abundance is a measure of how common or rare a species is relative to other species in a defined location or community (McGill et al., 2007). Analysis revealed that the relative species abundance under Felidae and Cervidae family has the highest individual $(n=17 \%)$ among
36 mammal species, followed by Sciuridae, Cercopithecoidae and Viverridae $(n=9 \%)$ and Hystricidae $(n=6 \%)$ respectively. While, Canidae, Herpestidae, Leporidae, Manidae, Melinae, Muridae, Mustelidae, Tupaiidae, Proboscidae, Pteromyidae, Suidae and Ursidae had lowest abundance in the study area $(n=3 \%)$ (Figure 2).

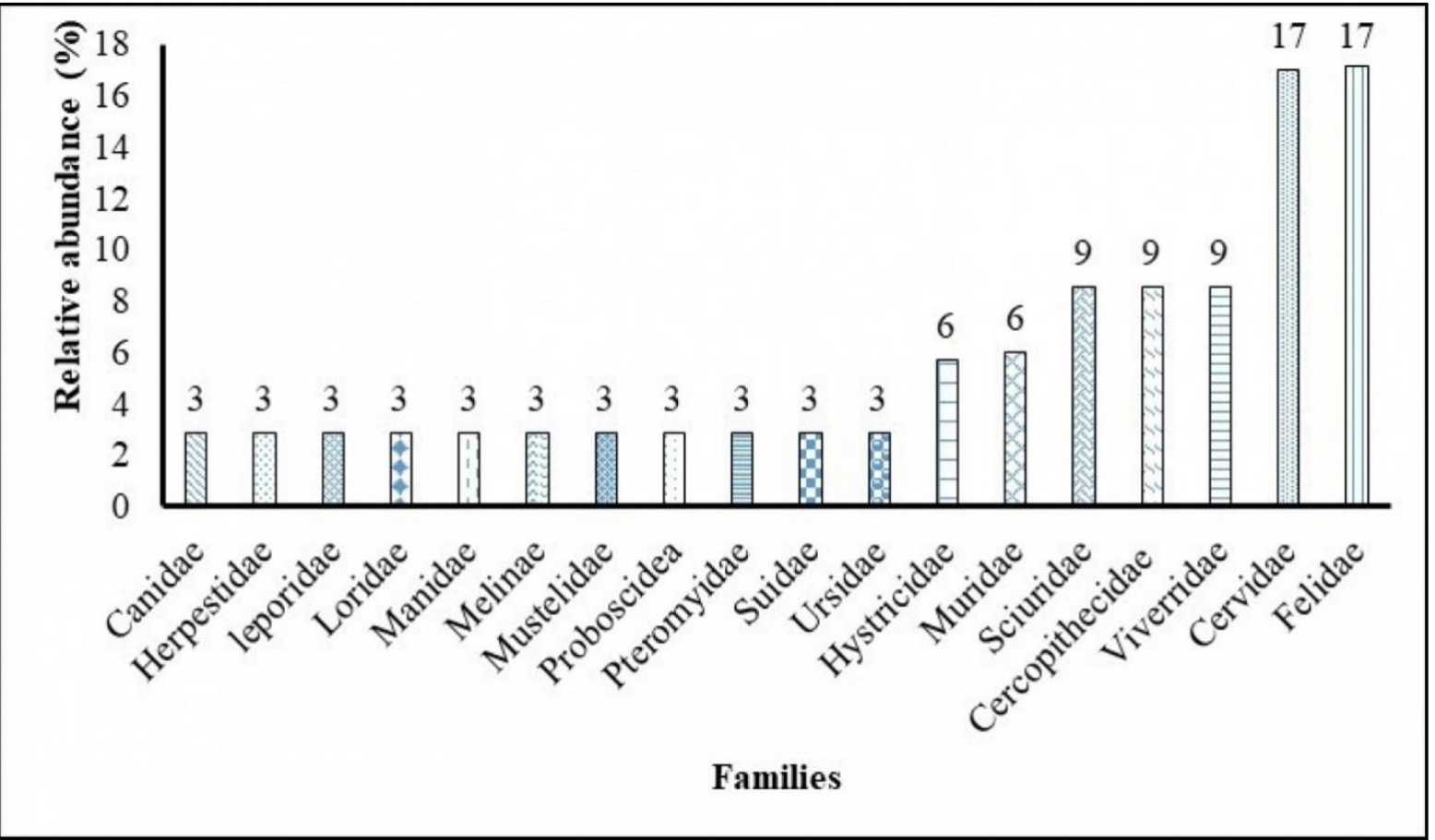

Figure 2. Relative species abundance of wildlife species (Families) under Sarpang district.

The highest records of felid species under Felidae in Sarpang could be due to the intact landscape connectivity with other ecologically riched protected areas of Bhutan such as RMNP, JSWNP, and PWS (Tenzin et al., 2019; Tenzin et al., 2021). Further, intact landscape connectivity and habitat contiguity also contributed to reporting six felid species from the southern central region of Sarpang (Tenzin et al. (2019) which is second to RMNP that had recorded eight felid species and declared as a Felid hotspot of Bhutan (Tempa et al.,2013; Tempa et al., 2019). However, felid abundance, density, and distribution from Sarpang district is still remain understudied, that requires separate study in the future.

The wide distribution of ungulates under the Cervidae family indicates that Sarpang district landscape can support more Felid species. Tempa (2017) and Thinley et al. (2018) had suggested that the widespread presence of wild ungulates indicates a healthy ecosystem that can support more Felid population, especially tigers and other wild cats' species in the ecosystem. The studies of Tempa (2017) and Tempa et al. (2019) had also substantiated that abundant availability of biggersized ungulates such as gaur (Bos gaurus Smith 1827) and sambar (Rusa unicolor Kerr, 1792) also shows strong determinant to tiger occupancy especially in the central part of Bhutan. However, a concern of depleting prey species has been raised, due to poaching which contributes to the declining predator population in the wild (Karanth and Gopal, 2005; Seidensticker, 2010; Ripple et al., 2014). Therefore, Divisional Forest Office at the district 
level must step-up patrolling efforts and to larger home ranges. Thus, most of the lessersurveillances using Spatial Monitoring and known species were under-represented (Dhendup Reporting Tools (SMART) Conservation software, and Dorji, 2018). Therefore, a separate study on across the landscapes to protect ungulates which in return will protect both bigger-sized \& small Felid species that will help in maintaining the vibrant ecosystem in the southern landscapes of Bhutan.

Meanwhile, the district has relatively captured less mammal species that belong to Canidae, Herpestidae, Leporidae, Manidae, Melinae, Muridae, Mustelidae, Tupaiidae, Proboscidae, Pteromyidae, Suidae, and Ursidae family. This indicates that species of these families have a less species diversity, unlike Felid and Cervidae. Nevertheless, most of the past camera traps survey was targeted only at large-sized carnivores (Tiger) and ungulates (elephants) with bigger grid sizes due small-sized mammal species with smaller grid sizes is suggested to capture more lesser-known species in future studies.

\section{Conservation Status of mammal species as per IUCN Redlist, CITES \& FNCAB (1995)}

The conservation status of mammal species present under Sarpang district were updated as per IUCN Red List for Threatened species (2019), CITES, and FNCAB (1995) for conservation purposes. Among 36 mammal species, 47\% $(n=17)$ of the species were categorized under LC, $22 \%$ NT $(n=8)$ and $14 \%$ VU $(n=5)$ and EN $(n=5)$ respectively, while, $3 \%(n=1)$ are categorized under CR (Figure $3 \&$ Table No. 3).

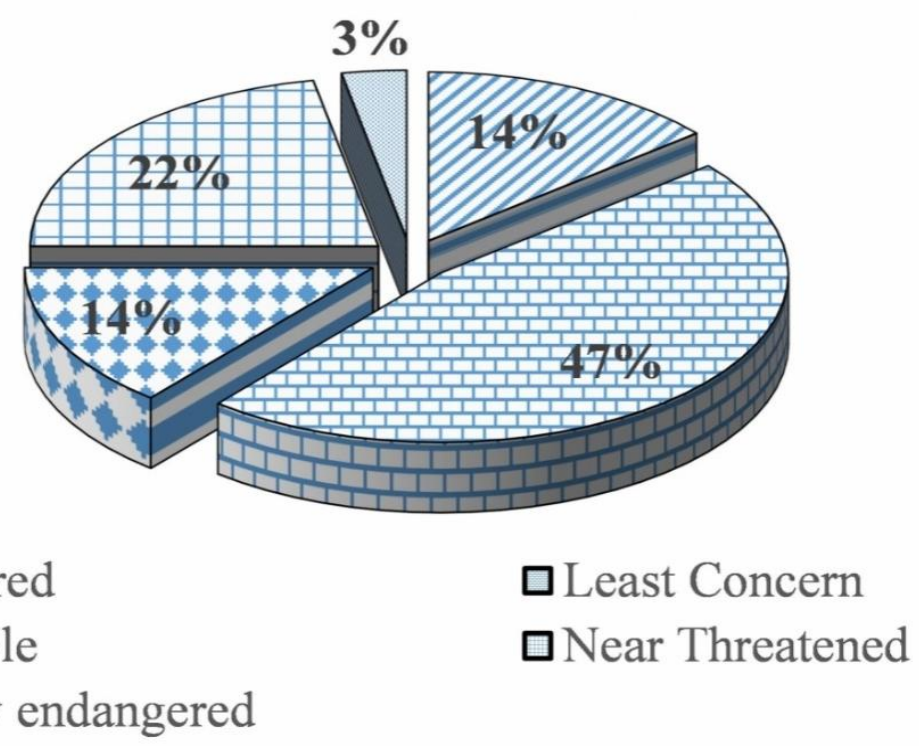

Figure 3. IUCN Conservation status of mammal species (\%) found in Sarpang district.

Among 36 mammal species, only 11 mammal species were appended under CITES Appendix I, seven in appendix II, and two in Appendix III respectively (Figure 4). Therefore, any international trades of CITES listed mammal species (appendix I, II \& III) must follow the specific legal obligation or international protocols before trading the mammal parts and derivatives to other countries (CITES, 1973). Likewise, only nine, among 23 mammal species were categorized under Schedule I which is legally protected under the FNCAB (1995) in Bhutan (Royal Government of Bhutan [RGoB], 1995). 


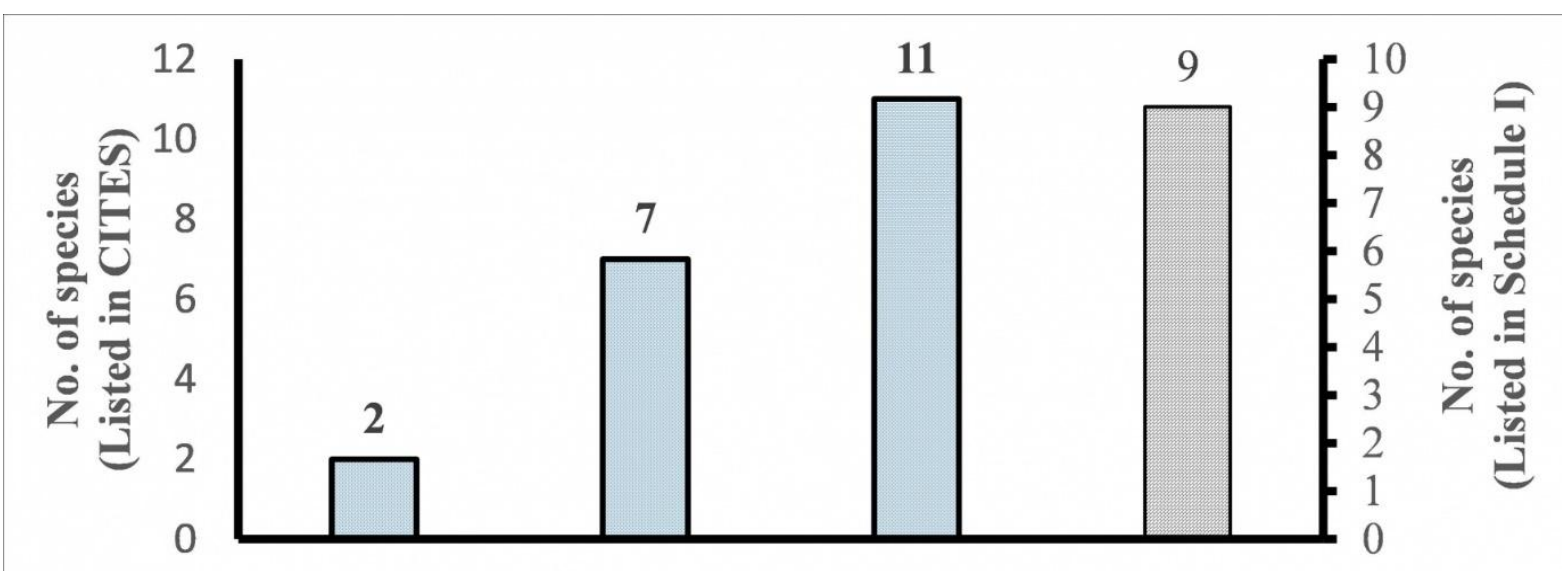

Appendix III Appendix II Appendix I Schedule I

\section{CITES Appendix \& Schedule I \\ 口CITES Appendix \\ $\square$ Schedule I of FNCAB}

Figure 4. Mammal species are listed in CITES Appendix and Schedule I of Forests and Nature Conservation Act of Bhutan (1995).

With regards to conservation status, more than $50 \%(n=19)$ of checklist species were globally threatened which requires high conservation priority, especially under Sarpang district. However, since the district being outside the protected areas (Non-Protected Area), protection and conservation of those threatened species will be challenging, solely due to the lack of a structured Division-based Conservation plan, unlike the protected areas which leads to haphazard allocation of timber resources (rural as well as commercials purposes) from the core wildlife habitats. Further, Dhendup and Dorji (2018) and Tenzin et al. (2019) also reported that although regulations exist in the Non-protected areas, wildlife species might be threatened, since Divisional Forest Offices (DFOs) does a lot of forest management, resource allocation (subsidized/ commercials), and other forestry-related public service deliveries. Thus, the recent DoFPS initiative in preparing Division Management Plans for 14 Divisional Forest Offices under International Climate Initiative [IKI] project (WWF Bhutan) and Biological Corridor Management plan (funded by Bhutan for Life Secretariat [BFL) will address the above issues \& secure the wildlife species under this landscapes in future.

On other hand, Bhutan has recorded more than 200 mammal species (Wangchuk et al., 2004), only 23 mammal species (10\%) are legally protected under Schedule I of FNCAB, 1995 (RGoB, 1995). Likewise, in the case of Sarpang

district, only nine mammal species which includes Tiger (Panthera trigris), Common leopard (Panthera pardus), Clouded leopard (Neofelis nebulosa), Leopard cat (Prionailurus bengalensis), Golden langur (Trachypithecus geei), Asian elephant (Elephas maxmus), Himalayan black bear (Ursus thibetanus laniger), Guar (Bos gaurus) and Himalayan serow (Capricornis sumatraensis) were legally protected under Schedule I of FNCAB (1995) irrespective of their conservation status in IUCN and CITES. However, most of the mammal listed in schedule I of FNCAB (1995) requires critical review and species validation especially the occurrence of Rhinoceros (Rhinoceros unicornis), Pigmy hog (Sus sylvanicus), and Hapid hare (Caprolagus hispidus) in Bhutan. Because there is no valid scientific evidence supporting the presence of those species except a few scanty anecdote reports existed before the 1990s. Further, both common name and scientific names for pangolin and leopard listed in schedule I were vague and inconsistent (i.e. need to specify which species of pangolin and leopard species is it?) that requires immediate updation by the DoFPS. Therefore, species listed in schedule I require immediate review and validation by the DoFPS to make a consistent name (common and scientific name) and accordingly update the lists of Schedule I species in revised FNCAB (2022) in the future. 


\section{CONCLUSION}

In summary, management of mammal diversity has become of utmost importance in light of rampant declining global mammal biodiversity due to anthropogenic activities, data deficiency, and climate change effects. Nevertheless, the availability of a structured mammal checklist in the PAs and NPA in Bhutan is the only solution to develop pragmatic conservation \& management plans which can ensure effective future conservation policies. Therefore, the urgent requirement of landscape-based species conservation plans (i.e. Division or district-based conservation and management plan) for 14 DFOs and BC-03; periodic monitoring of existing keystone and other lesser-known species using camera traps and urgent validation of name of Schedule I species were suggested for long-term conservation and management of wildlife species under the jurisdiction of Sarpang district in future.

\section{ACKNOWLEDGMENT}

The authors would like to acknowledge $\mathrm{Mr}$. Lobzang Dorji, Director, Department of Forests and Park Services (DoFPS) under the Ministry of Agriculture and Forests for his kind approval (Approval receipt No.237, dated 24th November 2020 vide letter No. SFD/RPES/RIU-03/20202021/358, dated 20th November 2020). Likewise, Ms. Karma Choki, Sr. Forestry Officer (CA|TS Project Focal), and Mr. Tshering Dorji, Sr. Forestry Officer (BFL Focal) are equally acknowledged for their support rendered during the course of the field survey. Besides these, the team also would like to thank Mr. Tashi Wangdi, SFR-I, Mr. Kezang Dhendup, SFR-I, Mr. Chencho Nidup, SFR-III, Mr. Tshering, FR-II, and Mr. Naten Tshering, Sr. Forester for data collection. Simultaneously, the authors also acknowledge IDA-World Bank and Bhutan Foundation for funding National Tiger Survey (2014-2015); WWF Bhutan for funding National Tiger Survey(2014-2015), Zero Poaching project (2016-2018), and CA|TS (2020) and Bhutan For Life Secretariat (BFL) project for funding RBA inside BC-03 in 2019 and finally to reviewers for refinement of the manuscript.

\section{REFERENCES}

Barlow, J., Lennox, G.D., Ferreira, J., Berenguer, E., Lees, A.C., Nally, R.M., Thomson, J. R., Ferraz, S.F.D.B., Louzada, J., Oliveira, V.H.F., Parry, L., Ribeiro De Castro Solar, R., Vieira, I.C.G., Arag ao, L.E.O.C., Begotti, R.A., Braga, R.F., Cardoso, T.M., de Oliveira, R.C., Souza, C.M., Moura, N.G., Nunes, S.S., Siqueira, J.V., Pardini, R., Silveira, J.M., Vaz-De-Mello, F.Z., Veiga, R.C.S., Venturieri, A., Gardner, T.A. (2016). Anthropogenic disturbance in tropical forests can double biodiversity loss from deforestation. Nature, 535, 144-147.

Butchart, S. H. M. et al. (2010). Global biodiversity: indicators of recent declines. Science, 1164-1168.

CITES. (1973). Text of Convention: Convention on International Trade in Endangered Species of Wild Fauna and Flora, CITES Secretariat, Geneva, Switzerland.

Dhendup, T., and Dorji, R. (2018). Camera-trap records of small carnivores from Gedu Territorial Forest Division, Bhutan. Small Carnivore. Conservation, 56, 1-6.

Dorji, D.P., \& Santiapillai, C. (1989). The Status, distribution and conservation of the tiger (Panthera tigris) in Bhutan. Biological Conservation, 48, 311-319.

DoFPS. (2015). Counting the Tigers in Bhutan: Report on the National Tiger Survey of Bhutan 2014 - 2015. Department of Forests and Park Services, Ministry of Agriculture and Forests. Kuensel Press.

Department of Agriculture [DOA]. (2012). Data collection survey on strategic Agricultural water supply and management in southern Bhutan (Final report of Japan International Cooperation Agency (JICA) Sanyu Consultants INC., Department of Agriculture, Thimphu, Bhutan.

Esmaeili, H.R., Mehraban, H., Abbasi, K., Keivany, Y., \& Brian, W.C. (2017). Review and updated checklist of freshwater fishes of Iran: Taxonomy, distribution and conservation status. Iranian Journal of Ichthyology, 4 (S1), 1-114.

FRMD. (2020). Forest Facts and Figures-2019. Forest Resource Management Division. 
Department of Forest and Park Services, Bhutan. Kuensel Press.

Jigme Khesar Strict Nature Reserve. (2020). Mammals of Jigme Khesar Strict Nature Reserve. Department of Forests and Park Services, Ministry of Agriculture and Forests, Haa, Bhutan. Kuensel Press.

Jomotsangkha Wildlife Sanctuary. (2018). A pictorial record of Mammal of Jomotsangkha Wildlife Sanctuary, Jomotsangkha, Department of Forests and Park Services, Bhutan. Kuensel Press.

Jones, G., Jacobs, D. S., Kunz, T. H., Willig, M. R. \& Racey, P. (2009). Carpe noctem: the importance of bats as bioindicators. Endangered Species Research, 8, 93-115.

Jones, K.E., and Safi, K. (2011). Ecology and evolution of mammalian biodiversity. Phil. Trans. R. Soc. B, 366, 2451-2461.

Karanth, K. U., \& Gopal, R. (2005). An ecologybased policy framework for human-tiger coexistence in India. Conservation Biology Series-Cambridge, 9, 373.

Keesing, F., Belden, L.K., Daszak, P., Dobson, A., Harvell, C.D., Holt, R.D., Hudson, P., Jolles, A., Jones, K.E., Mitchell, C.E., Myers, S.S., Bogich, T., \& Ostfeld, R.S. (2010). Impacts of biodiversity on the emergence and transmission of infectious diseases. Nature, $468,647-652$.

Koirala, B. K., and Jamtsho,Y. (2019). Faunal Diversity of Jigme Dorji National Park- A Photographic Guide. Department of Forests and Park Services, Royal Government of Bhutan. Kuensel Press.

Kunz, T. H., Braun de Torrez, E., Bauer, D., Lobova, T. \& Fleming, T. H. (2011). Ecosystem services provided by bats. ANNALS of the New York Academic of Science, 1223, 1-38.

Mace, G. M. et al. (2005). Biodiversity. In Ecosystems and human well-being, vol. 1 (eds R. Hassan, R. Scholes \& N. Ash), pp. 53-98. Washington, DC: Millennium Ecosystem Assessment.

McGill, B. J., Etienne, R. S., Gray J. S., Alonso, D., Anderson, M. J., Benecha, H. K., Dornelas, M., Enquist, B. J., Green, J. L., He, F., Hurlbert, A. H., Magurran, A. E., Marquet, P. A., Maurer, B. A., Ostling, A., Soykan, C.
U., Ugland, K. I., \&White, E. P. (2007). Species abundance distributions: moving beyond single prediction theories to integration within an ecological framework. Ecology Letters, 10, 995-1015.

McDougal, C.W., \&Tshering, K. (1998). Tiger Conservation Strategy for the Kingdom of Bhutan. Thimphu: Nature Conservation Division, Department of Forests, Bhutan. Kuensel Press.

Ministry of Work \& Human Settlement. (2019). Flood hazard assessment for Sarpang Dzongkhag. Flood Engineering and Management Division, Department of Engineering Services, Ministry of Works and Human Settlement, Thimphu, Bhutan. Kuensel Press.

Mitermeier, R.A., Gil, P.R, Hofmann, M.., Pilgrims, J, Brooks, T., Mitermeier, C.G., Lamoreux, J., \& Da Fonseca, G.A.B. (2004). Hotspots Revisited: Earth's Biologically Richest and Most Endangered Terrestrial Ecoregions. CEMEX, USA.

National Biodiversity Centre. (2017). Biodiversity Statistics of Bhutan: A Preliminary Baseline, National Biodiversity Centre, Ministry of Agriculture and Forests, Thimphu, Bhutan. Kuensel Press.

NEC. (2011). Biodiversity. National Environment Commission, Thimphu, Bhutan, Kuensel Press.

National Statistics Bhutan [NSB]. (2018). Population \& Housing census of Bhutan 2017: Sarpang Dzongkhag. National Statistics Bureau, Thimphu, Bhutan.

Olson, D.M., \& Dinerstein, E. (2002). The Global 200: Priority ecoregions for global conservation. Annals of the Missouri Botanical Garden, 89 (2),199-224.

Ohsawa, M. (1987). Life zone ecology of the Bhutan Himalaya, Laboratory of Ecology, Chiba University, Japan.

Penjor, U., MacDonald, D.W., Wangchuck, S., Tandin, T., \&Tan, C.K.W. (2018). Identifying important conservation areas for the Clouded Leopard Neofelis nebulosa in a mountainous landscape: Inference from spatial modeling techniques. Ecology and Evolution, 8, 4278-4291. 
Penjor, U., Wangdi, S., Tandin, T. \& David W. Macdonald, D.W.(2021). Vulnerability of mammal communities to the combined impacts of anthropic land-use and climate change in the Himalayan conservation landscape of Bhutan. Ecological Indicator, 121,107085 .

Powers, R.P. and Jetz, W. (2019). Global habitat loss and extinction risk of terrestrial vertebrates under future land-use-change scenarios. National Climate Change, 9, 323329.

PWS. (2019). A Mammal Checklist of Phibsoo Wildlife Sanctuary: A preliminary listing. Phibsoo: Phibsoo Wildlife Sanctuary, Department of Forests and Park Services, Ministry of Agriculture \& Forests, Sarpang, Bhutan. Kuensel Press.

RGoB. (1995). The Forests and Nature Conservation Act of Bhutan, 1995, Department of Forests and Park Services, Ministry of Agriculture, Bhutan. Kuensel Press.

RGoB. (2008). The constitution of the Kingdom of Bhutan. Kuensel Press.

Ripple, W.J., Estes, J.A., Beschta, R.L., Wilmers, C.C., Ritchie, E.G., Hebblewhite, M., Berger, J., Elmhagen, B., Letnic, M., \& Nelson, M.P. (2014). Status and ecological effects of the world's largest carnivores. Science, 343, 1241484.

Sanderson, J., and Harris, G. (2012). Automatic data organization, storage, and analysis of camera trap pictures. Journal of Indonesia Natural History, 1 (1), 11-19.

Schipper, J. et al. (2008). The status of the World's land and marine mammals: diversity, threat and knowledge. Science, 322, 225-230.

Seidensticker J., 2010. Saving wild tigers: a case study in biodiversity loss and challenges to be met for recovery beyond 2010. Integrative Zoology, 5, 285-299.

Tempa, T., Hebblewhite, M., Mills, L.S., Wangchuk, T.R., Norbu, N., Wangchuk, T., Nidup, T., Dendup, P., Wangchuk, D., Wangdi, Y., \& Dorji, T. (2013). Royal Manas National Park, Bhutan: a hot spot for wild felids. Oryx, 47(2), 207-210.

Tempa, T. (2017). The ecology of montane Bengal tigers (Panthera tigris tigris) in the
Himalayan Kingdom of Bhutan. Scholar Works at University of Montana Graduate Student Theses, USA, Montana.

Tempa, T., Hebblewhite, M., Goldberg, J.F., Norbu, N., Wangchuk, T.R., Xiao, W., \&Mills, S.L. (2019). The spatial distribution and population density of tigers in mountainous terrain of Bhutan. Biological Conservation, 238,108192 .

Tenzin, J., \& Dhendup, P. (2017). Habitat Characteristics, Relative Abundance and Conservation Threats of Himalayan Bull Frogs (Nanorana leibigii Günther, 1860) in Primary Tributaries of Simkhar River, Bhutan. Bhutan Journal of Natural Resources \& Development, 4, 29-38.

Tenzin, J.; Dhendup, T., Dhendup, P., Dorji, T., Choki, K., Wangchuk, S., Dorji, S., Nidup, C., \& Dorji, T. (2019). Six felid species occur outside protected areas in south-central Bhutan, Catsnews, 70, 25-27.

Tenzin, J., and Wangyal, J.T. (2019). New record of Blue-eyed Eastern Spadefoot Toad Leptobrachium bompu (Amphiia: Megophryidae) from Sarpang District in Bhutan. Journal of Threatened Taxa,11: 13385-13389.

Tenzin, J., Wangdi, T., Dhendup, K., Nidup, C., Tshering, D., Chophel., Penjor S., Phuntsho Y., Drukpa, D., Wangdi, Y., \& Tshering, L. (2021). Rapid Biodiversity Assessment: survey report of mammal and bird's species inside the Biological Corridor (03) under Sarpang-Tsirang Forest Division, Department of Forests and Park Services, Ministry of Agriculture and Forests, Bhutan.

Tenzin, J., Dhendup, P., Choki, K., Wangdi, T., Tshering, D., Dorji, D., Bomzan, M., \&Tenzin K., Thinley P. (2021). Increased tiger numbers in Sarpang Forest Division in south central Bhutan. Catnews 73: 27-28.

Thinley, P., Norbu, T., Rajaratnam, R., Vernes, K., Dhendup, P., Tenzin, J., Choki, K. Wangchuk, S., Wangchuk, T., Wangdi, S., Chhetri, D.M., Powrel, R.B., Dorji, K., Rinchen, K., \& Dorji, N. (2019). Conservation threats to the endangered golden langur (Trachypithecus geei, Khajuria 1956) in Bhutan. Journal of Primatology, 61 (2), 257-266. 
Wangchuk, T., Thinley, P., Tshering, K., Tshering, C., Yonten, D., Pema, B., \& Wangchuk, S., (2004). Field Guide to the mammals of Bhutan. Department of Forests and Park Services, Ministry of Agriculture, Royal Government of Bhutan. Kuensel Press. 